\title{
Initial Real-Life Experience from a Designated COVID-19 Centre in Athens, Greece: a Proposed Therapeutic Algorithm
}

\author{
Ioannis G. Baraboutis ${ }^{1}$ (D) Panagiotis Gargalianos ${ }^{2} \cdot$ Eleni Aggelonidou $^{1} \cdot$ Andreas Adraktas $^{1} \cdot$ Collaborators
}

Accepted: 13 May 2020 / Published online: 26 May 2020

(C) Springer Nature Switzerland AG 2020

\begin{abstract}
We report our initial experience with the management of a mixed group of patients with COVID-19 infection, admitted and treated in a designated COVID-19 centre in the Athens Metropolitan area over a 4-week period. The SARS-CoV-2 pandemic presented a huge challenge to the Greek National Healthcare System and healthcare workers. Their response so far has been miraculously effective. Since there are essentially no therapeutic guidelines yet for this disease, we relied mainly on our medical intuition, our empathy for our patients and team work to do the best possible for 49 people with this infection. We present the therapeutic algorithm we gradually developed (on the job) and applied in our patients, based on continuous creative brainstorming and monitoring of the literature.
\end{abstract}

Keywords COVID-19 infection · Coinfections · Bovine coronavirus · Ferritin · C-reactive protein · D-dimer · Viral replication · Human immunodeficiency virus (HIV) - Pneumocystis jiroveci pneumonia · Oral candidiasis · Adult respiratory distress syndrome · Cytokine release syndrome · Immunomodulatory therapy · Interleukin- 6 - Interleukin- 1 Soluble urokinase-type plasminogen activator receptor $\cdot$ Antimicrobial therapy $\cdot$ Corticosteroids $\cdot$ Lung microbiota

\section{Introduction}

The SARS-CoV-2 pandemic found Greece at a phase of attempting to exit a 10 -year socioeconomic crisis. The National Health Care System has greatly suffered the consequences of this crisis and is soon reaching a critical point. A large body of physicians is about to retire simultaneously

This article is part of the Topical Collection on COVID-19

Ioannis G. Baraboutis

ioannisbaraboutis@yahoo.gr; i-baraboutis@pammakaristos-hosp.gr

Panagiotis Gargalianos

pagargalianos@gmail.com

Eleni Aggelonidou

eleni_agelonidou@yahoo.com

Andreas Adraktas

andreas.adraktas@gmail.com

1 Internal Medicine Clinic, Pammakaristos Hospital of Divine Providence (Reference Centre for COVID-19 Infection), Iakovaton 43, 11144 Athens, Greece

2 Internal Medicine and Infectious Diseases Department, Hellenic Society for Infectious Diseases, Athens Medical Centre/Athens Medical Group, Distomou 5, 15125 Marousi, Greece while a lot of young doctors and nurses have sought work and education outside the country. Uncertainty and fear have been spreading all over the country, especially after witnessing the situation in Italy.

\section{Methods}

Our small hospital in Athens Metropolitan area (capacity of 170 beds, actual capacity based on staff levels 60 beds) was designated as a COVID-19 centre, aiming to treat up to 'medium' severity of patients with COVID-19 infections, as we lack intensive care unit beds but do have 2 high-dependency unit beds. Before the pandemic, our hospital was facing significant staff shortages and a large part of the medical equipment was relatively old. It should be noted that computed tomography was not available in our hospital during the period of this report. Our personnel had to be trained through fasttrack courses, as previous collective experience with personal protective equipment (PPE) had been relatively limited in our country. Our personnel initially exhibited a significant degree of anxiety and tension, something totally expected in the face of a pandemic without available therapeutic guidance, not only locally but even worldwide (https://www.who.int/). 
To respond to the challenge, we had to use all our collective clinical experience, our empathy for our patients and our sense of camaraderie. A pandemic has been repeatedly paralleled with a war situation, where unconventional methods may need to be used to prevent huge losses, especially at the beginning of the pandemic, where minimal data are available. Besides our collective clinical experience, including the one from the HIV/AIDS epidemic, we even had discussions with senior veterinarians, who are familiar with bovine coronaviruses. Their input and pertinent literature about a relatively high rate of coinfections in syndromes caused by bovine coronaviruses did influence our practice [1]. We obviously had to combine their paradigm and approach with continuous literature review of COVID-19 treatment data, our evolving national guidelines, extreme caution for side effects and frequent audit of our practice.

We overall treated a total of 49 patients with COVID-19 infection over a 4-week period, March 16 through April 12, 2020 (see Table 1 for demographics). Patients were either admitted from home or transferred from another tertiary care hospital for continued care. The median age was 63 years and the age range was 20-95 years. In case of need for intubation, the patient was subsequently transferred to an ICU bed elsewhere. We gradually developed a 'relatively clear' therapeutic algorithm, presented below.

\section{Results}

Based on our national and also international guidelines, we would start treatment with hydroxychloroquine and azithromycin (https://eody.gov.gr/wp-content/uploads/2020/ 03/covid-19-algorithmos-therapeia.pdf; https://www.ema.

Table 1 Demographics, treatment details and outcome

$\begin{array}{ll}\text { Patient number } & 49 \\ \text { Men/women } & 30 / 19 \\ \text { Median age } & 30 / 19 \\ \text { Median duration of stay } & 14 \text { days } \\ \text { Azithromycin } & 48 / 49 \\ \text { Hydroxychloroquine } & 46 / 49 \\ \text { 3rd agent for community-acquired pneumonia } & 33 / 49 \\ \text { Escalated regime } & 22 / 49 \\ \text { 'PJP' regime } & 8 / 49 \\ \text { Corticosteroids } & 11 / 49 \\ \text { PaO2/FiO2 ratio less than 200 } & 12 / 49 \\ \text { Intubation } & 8 \\ \text { Probable cytokine release syndrome } & 3 \\ \text { Total deaths } & 6 \\ \text { Deaths directly attributed to COVID-19 infection } & 3\end{array}$

europa.eu/en/news/update-treatments-vaccines-againstcovid-19-under-development). We realised that quite a few women and younger men did not need more than that. Some of those patients could have probably required just azithromycin, a unique broad-spectrum antimicrobial with long intracellular half-life combined with anti-inflammatory properties, or maybe nothing at all. We avoided using hydroxychloroquine in patients with known G-6-PD deficiency, ischaemic heart disease with or without heart failure and macular degeneration (https://newsroom.heart.org/news/ caution-recommended-on-covid-19-treatment-withhydroxychloroquine-and-azithromycin-for-patients-withcardiovascular-disease-6797342).

In case of intolerance or allergy to macrolides, we started using a similar unique class of antimicrobials, i.e. tetracyclines, most often doxycycline [2].

Overall, 46/49 patients received hydroxychloroquine (2 had known G-6PD deficiency and one with known macular degeneration) and 48/49 received azithromycin.

For more unwell patients on admission, especially with high fever, elevated C-reactive protein (CRP) and ferritin and lobar or multi-lobar infiltrates in addition to the 'usual' COVID-19-related interstitial pattern, we used on admission an additional antimicrobial agent approved for communityacquired pneumonia, for example amoxicillin with or without clavulanate or ceftriaxone.

For patients with significant penicillin allergies, we frequently elected to use teicoplanin due to in vitro data about possible direct antiviral activity, already published experience in patients with COVID-19 infection and low rate of adverse effects [3]. We noted that quite a few patients of various ages and both genders with diabetes mellitus seemed to be in need of such a regime and, thankfully, most of them responded well [4].

Overall, 33/49 patients received a 3rd agent for community acquired pneumonia.

We noticed that, despite the regimes used above, a few of our patients exhibited clinical, laboratory and radiological deterioration, most of them between days 7 and 12 from onset of symptoms. They exhibited worsening $\mathrm{PaO} 2 / \mathrm{FiO} 2$ ratios, worsening interstitial infiltrates and/or superimposed lobar or multi-lobar infiltrates, indicating possibly a bacterial superinfection. Interestingly, we rarely noticed accompanying hypotension requiring fluid resuscitation or inotropic support, despite continuing most of the patients' prior medications, especially angiotensin-converting enzyme inhibitors (ACEI) or angiotensin II receptor blockers (ARB). Even though we did sent quite a few culture specimens (blood, and sputum) as well as urine pneumococcal and Legionella antigen in many patients, we essentially did not detect bacterial co-pathogens, except for a few instances of Candida in the sputum, which is frequently viewed as contaminant. 
However, based on the veterinarian paradigm and lack of proven alternative therapeutic guidance or strategy for the deteriorating patient with COVID-19 infection, we started deploying a careful antimicrobial regime escalation strategy on those patients.

We used an 'escalated' regime that included broad spectrum agents for healthcare-associated pneumonia, tailored to our local susceptibility data. Examples were either piperacillin-tazobactam or a carbapenem, combined with teicoplanin or linezolid. We did use tigecycline in 1 patient, although one needs to monitor fibrinogen levels after a few days of treatment [5].

In a few patients, we ended up using the 'escalated' regime combined with corticosteroids, when the $\mathrm{PaO} 2 / \mathrm{FiO} 2$ ratio dropped to 200 or lower but without 'significant' lymphopenia (see below).

Additionally, for people with 'significant' lymphopenia, which we defined as a total lymphocyte count of 850 per $\mathrm{mm}^{3}$ or lower (back in the AIDS era, this roughly corresponded to a CD4 count of 150 cells per $\mathrm{mm}^{3}$ ) and respiratory deterioration with a $\mathrm{PaO} 2 / \mathrm{FiO} 2$ ratio of 200 or lower, we empirically used, for a few days, what used to be the treatment for Pneumocystis jiroveci pneumonia (PJP) in the AIDS era, i.e. high-dose trimethoprim-sulfamethoxazole and corticosteroids [6]. Trimethoprim-sulfamethoxazole is advantageous as a broad spectrum antibacterial and antifungal agent, while some believe it also possesses direct antiviral and anti-inflammatory properties [7].

Two of our patients presented on days 7-10 of symptoms with 'unexpected' oral candidiasis (no risk factors like known immunosuppression or recent antimicrobial use). Interestingly, one of them had symptoms compatible with oesophageal candidiasis, which in our view was indicative of a more profound and acute $\mathrm{T}$ cell immunologic deficit, already described in the literature [8]. We have not come across yet of other similar reports in the literature.

We used corticosteroids at a dose of $4 \mathrm{mg}$ of dexamethasone every $6 \mathrm{~h}$ for non-diabetics and $8 \mathrm{mg}$ every $6 \mathrm{~h}$ for people with diabetes mellitus for $48-72 \mathrm{~h}$ and then started rapid tapering.

Overall, 22/49 patients received the 'escalated' regime, 8 patients received trimethoprim-sulfamethoxazole and 11 patients received corticosteroids.

Out of the 49 patients we treated, we had 6 deaths. Three patients, ages 90, 92 and 95, died from healthcare-associated pneumonia rather than pneumonia due to COVID-19 infection and they were not intubated. Two patients died from likely cytokine release syndrome, which we attempted to treat with corticosteroids only, as immunomodulatory therapies were not available at that time. A total of 8 patients had to be intubated, 7 of them were transferred to another hospital (Table 1).

\section{Discussion}

Overall, we ended up using the 'escalated' regime in nearly $40 \%$ of our patients, in some of them with corticosteroids, and additionally the 'PJP' regime in nearly one-fourth of our patients who 'typically' deteriorated due to COVID-19 infection. For the patients where the enhanced regimes were used, we did notice clinical response/improvement within 2-3 days and even significant radiological improvement within 45 days, something not usually observed with 'classic' community- or healthcare-acquired pneumonia. Most of the antimicrobial courses we used were for 5-7 days.

As mentioned, we recovered essentially no co-pathogens from the microbiological investigations we sent in our small sample of patients. One would argue that we adopted an unconventional and risky strategy and have been systematically violating the classic rule of traditional General Medicine and Infectious Diseases teaching, i.e. 'antimicrobials are not recommended for a viral infection'.

However, the following points are worth mentioning:

We observed a consistent pattern of progressive CRP and ferritin elevation, along with worsening $\mathrm{PaO} 2 / \mathrm{FiO} 2$ ratio and new infiltrates, either interstitial or - even more-lobar/multilobar in patients who clinically deteriorated at days $7-10$, as already described. We cannot recall many viral infections known to increase the CRP and ferritin in such high levels and additionally cause lobar and multi-lobar infiltrates. When those patients were administered antimicrobials with or without corticosteroids, the timing of their clinical and laboratory improvement was very similar to the timing of response to a 'classic' bacterial pneumonia, in terms of improved $\mathrm{PaO} 2 /$ FiO2 ratio, decreased polymorphonuclear white blood cells, increased lymphocytes and decreasing $\mathrm{C}$-reactive protein (about ferritin, see below).

We essentially had minimal antimicrobial-related side effects attributed to our strategy. We had 1 case or oral candidiasis and 1 case of teicoplanin-related skin rash. Both happened after day 5 of antimicrobial therapy and while patients were improving otherwise. Quite a few patients had diarrhoea; however, in only 1 elderly female patient, it looked to be related to antimicrobial use and easily went away with discontinuation. There were no cases of Clostridioides difficile infection and no superinfections by multi-resistant healthcarerelated pathogens, even though all our patients stayed inhospital at least 2 weeks (median duration of stay was 2 weeks) and there was definitely blending with patients that were admitted from other hospitals and expected to be colonised by multi-resistant pathogens. In regard to the last argument, one could argue this could be partially attributed to higher quality than usual infection control precautions and measures.

Needless to say, we meticulously reviewed our antimicrobial choices on a daily basis, based on widely accepted principles of antimicrobial stewardship in regard to de-escalation, 
rationalisation and total duration of treatment [9]. As already mentioned, and based on our small experience so far, patients may not need more than 5 days of antimicrobial treatment per episode of deterioration, even the ones who have developed adult respiratory distress syndrome.

In terms of corticosteroids at the stage of clinical deterioration, our experience was that it was safe to give along with broad spectrum antimicrobials and we used them at a dose of $4 \mathrm{mg}$ of dexamethasone every $6 \mathrm{~h}$ for non-diabetics and $8 \mathrm{mg}$ every $6 \mathrm{~h}$ for people with diabetes mellitus. The reason for the chosen higher dose in diabetics was mostly theoretical and based on the association described between type 1 diabetes mellitus and adrenal insufficiency [10]. We did not have significant hyperglycaemia issues with that practice.

We believe we successfully prevented intubation with the above regimes in quite a few patients, including rapidly deteriorating high-risk patients, i.e. men in their $60-80$ s. We perfectly understand the risks of giving corticosteroids in patients with dropping lymphocyte counts; however, our initial experience showed that corticosteroids, combined with antimicrobials, not only were effective in slowing down ARDS in COVID-19 infection but additionally that the lymphocyte count promptly rose as soon as we initiated fast tapering [11].

There is growing experience on the beneficial role of immunomodulatory therapies for deteriorating patients, especially for the acutely and unexpectedly deteriorating patients with the cytokine release syndrome (CRS) [12]. In our experience, intense and prolonged rigours (2-3 days) around days 7-10 from symptom onset should alert the clinician to the possibility of this syndrome and trigger measurements of biologic markers like interleukin-6, interleukin-1 and, if available, other markers of endothelial activation like soluble urokinasetype plasminogen activator receptor (sUPAR). In our country, at this stage, these measurements and immunomodulatory treatments are not available outside research protocols [13]. If those treatments are not available, high-dose steroids might be effective and could be used in analogy to the haematology/ oncology literature on CRS treatment [14].

We have been following CRP and ferritin as inflammatory markers [6]. Our experience so far indicated that these 2 parameters follow different kinetics and our initial impression and working hypothesis were that, while CRP is mainly a marker of a coexistent infection or superinfection, ferritin might be a better marker of viral replication besides being a coinfection/superinfection marker. We realise that this is just a hypothesis that remains to be confirmed through appropriate large-scale studies. We have definitely noticed asymptomatic increases in ferritin that coincide temporally with positive polymerase chain reaction (PCR) testing.

We obviously do not have a pathophysiologic basis for treating a viral infection with antimicrobials, besides the veterinarian 'equivalent', if there is one yet. Last few years, there has been a growing number of reports on the role of lung microbiota in lung infection, disease susceptibility and severity. Alterations of lung microbiota could potentially modify immune response against SARS-CoV-2, the 'original' viral pathogen and secondary bacterial infection(s). In one study where metatranscriptome sequencing was conducted on bronchoalveolar lavage fluid (BALF) samples from 8 subjects with COVID-19, high evolution rate of the virus was noted. Meanwhile, no specific microbiota alteration in the BALF of COVID-19 patients was found in comparison to communityacquired pneumonia patients with other suspected viral causes [15].

\section{Conclusion}

We employed a stepwise antimicrobial strategy for our deteriorating patients with COVID-19 infection, based on our prior diverse clinical experience, the continuous audit of our practice, our collective sense of urgency, responsibility and creative intuition. Hospitalised patients with COVID-19 infection need comprehensive, meticulous and personalised care [16]. For example, it is well known by now that aggressive hydration should be avoided in these patients and keeping them on the 'dry side' is preferred; we routinely use diuretics to most of our patients [17]. Another crucial point is particular attention to the guidelines on appropriate deep vein thrombosis prophylaxis. In a few patients who deteriorated, especially ones with higher body mass index (BMI) and/or D-dimers more than 4-5 times the upper normal limit, we have used therapeutic doses of low molecular weight heparin until they recovered [18]. We have not had significant bleeding complications attributed to our practice.

We have been vigilant for problems like acute coronary syndromes, pulmonary embolism and/or exacerbation of prior diseases, depending on each patient's profile.

We have now started an outpatient Internal Medicine Clinic specifically for follow-up of COVID-19 patients recently admitted to our hospital [19].

\section{Limitations}

At this stage of the pandemic in our country and the circumstances of our practice, we had neither the numbers to prove our largely empirical therapeutic strategy practices are effective nor the time and capacity to set up a proper study, even though we are not sure what the term 'proper study' would mean to different people in a pandemic. Classic medical teaching emphasises how essential the medical diagnosis is for the patient so appropriate and individualised treatment can be initiated. Paradoxically, in COVID-19 infection, the diagnosis is obvious, a viral infection; however, treatment is lacking and is more than urgently needed as people die from this viral 
infection who were not supposed to. Maybe we do not have the 'whole' diagnosis or there are 'more than one' diagnoses in some patients. Our humble goal was just to communicate our collective institutional experience so far, as things are evolving [20]. Thankfully, we have not had significant side effects or superinfections attributed to the antimicrobial strategies or other practices we have implemented so far 'beyond' the existent guidelines and norms. We believe we have saved some lives by now and intend to continue refining our practice, based on more incoming literature data.

Acknowledgments Charalampos A. Thanos charalampos.a. thanos@gmail.com, Danai N. Mparlampa danai_mp@hotmail.com, Alexandra M. Giannou, alyannou@outlook.com.gr, Mahmoud M. Abdelrasoul mahmabdel@gmail.com, Alexandra K.S. Papadopoulou kkp1216@yahoo.gr, Ibraheem Aqeel ibraheemaqeel@yahoo.com, Konstantinos I. Alimissis tatewaffenss@yahoo.gr, Angeliki M. Chasouraki angchasouraki@gmail.com, Anastasios S. Chatziantoniou Huicho194@gmail.com, Vassiliki P. Patsoura bikipatsoura@yahoo.gr, Odysseas A. Violetis Odysseas.violetis@gmail.com, Louisa E. Tsagkaraki louisatsag@gmail.com, Christina D. Dimitriou Chrisdeme1105@gmail.com,Eleonora G. Chalatova Eleonora1976savv@gmail.com.

Author's Contributions IB: conceptualised the study design, reviewed and analysed the data, gradually developed the proposed algorithm, wrote the manuscript, critically reviewed and revised the manuscript and gave final approval of the version to be published.

PG, EA, AA: contributed to the development of the proposed algorithm through productive brainstorming.

Collaborators (total of 14): contributed to patient care and data collection. The corresponding author confirms that all collaborators have agreed to be included in a separate list/annex.

\section{References}

1. Saif LJ. Bovine respiratory coronavirus. Vet Clin North Am Food Anim Pract. 2010;26(2):349-64. https://doi.org/10.1016/j.cvfa. 2010.04.005.

2. Sodhi M, Etminan M. Therapeutic potential for tetracyclines in the treatment of COVID-19. Pharmacotherapy. 2020;40:487-8. https:// doi.org/10.1002/phar.2395.

3. Baron SA, Devaux C, Colson P, Raoult D, Rolain JM. Teicoplanin: an alternative drug for the treatment of COVID-19? Int $\mathrm{J}$ Antimicrob Agents. 2020;13:105944. https://doi.org/10.1016/j. ijantimicag.2020.105944.

4. Maddaloni E, Buzzetti R. Covid-19 and diabetes mellitus: unveiling the interaction of two pandemics. Diabetes Metab Res Rev. 2020;31:e33213321. https://doi.org/10.1002/dmrr.3321.

5. Brandtner A, Bachler M, Fries D, Hermann M, Ruehlicke J, Fux V, et al. Tigecycline interferes with fibrinogen polymerization independent of peripheral interactions with the coagulation system. Antibiotics (Basel). 2020;9(2):E84. https://doi.org/10.3390/ antibiotics 9020084 .
6. Cockerill FR 3rd, Edson RS. Trimethoprim-sulfamethoxazole. Mayo Clin Proc. 1987;62(10):921-9.

7. Stebbing J, Phelan A, Griffin I, Tucker C, Oechsle O, Smith D, et al. COVID-19: combining antiviral and anti-inflammatory treatments. Lancet Infect Dis. 2020 Apr;20(4):400-2. https://doi.org/10.1016/ S1473-3099(20)30132-8.

8. Chen $\mathrm{G}, \mathrm{Wu} \mathrm{D}$, Guo $\mathrm{W}$, et al. Clinical and immunologic features in severe and moderate coronavirus disease 2019. J Clin Invest. 2020: 137244. https://doi.org/10.1172/JCI137244.

9. Stevens MP, Patel PK, Nori P. Involving antimicrobial stewardship programs in COVID-19 response efforts: all hands on deck. Infect Control Hosp Epidemiol. 2020;13:1-2. https://doi.org/10.1017/ice. 2020.69.

10. Brož J, Urbanová J, Halčiaková K, Nunes MA, Brunerová L. Commentary: Early clinical indicators of Addison's disease in adults with type 1 diabetes: a nationwide, observational, cohort study. Front Endocrinol (Lausanne). 2019;10:456. https://doi.org/ 10.3389/fendo.2019.00456 eCollection 2019.

11. Zha L, Li S, Pan L, Tefsen B, Li Y, French N, et al. Corticosteroid treatment of patients with coronavirus disease 2019 (COVID-19). Med J Aust. 2020;212:416-20. https://doi.org/10.5694/mja2. 50577.

12. Mehta P, McAuley DF, Brown M, Sanchez E, Tattersall RS, Manson JJ, et al. COVID-19: consider cytokine storm syndromes and immunosuppression. Lancet. 2020. https://doi.org/10.1016/ S0140-6736(20)30628-0 No abstract available.

13. Efficiency in management of organ dysfunction associated with infection by the novel sars-cov- 2 virus through a personalized immunotherapy approach: the ESCAPE clinical trial; EudraCt number: 2020-001039-29.

14. Shimabukuro-Vornhagen A, Gödel P, Subklewe M, Stemmler HJ, Schlößer HA, Schlaak M, et al. Cytokine release syndrome. J Immunother Cancer. 2018;6(1):56. https://doi.org/10.1186/ s40425-018-0343-9.

15. Shen Z, Xiao Y, Kang L, et al. Genomic diversity of SARS-CoV-2 in coronavirus disease 2019 patients. Clin Infect Dis. 2020:ciaa203. https://doi.org/10.1093/cid/ciaa203 Published online 2020 Mar 9.

16. $\mathrm{Li} \mathrm{T}, \mathrm{Lu} \mathrm{H}$, Zhang W. Clinical observation and management of COVID-19 patients. Emerg Microbes Infect. 2020;9(1):687-90. https://doi.org/10.1080/22221751.2020.1741327.

17. Ling L, So C, Shum HP, et al. Critically ill patients with COVID-19 in Hong Kong: a multi centre retrospective observational cohort study. Crit Care Resusc. 2020;6.

18. Tan CW, Low JGH, Wong WH, Chua YY, Goh SL, Ng HJ. Critically ill COVID-19 infected patients exhibit increased clot waveform analysis parameters consistent with hypercoagulability. Am J Hematol. 2020. https://doi.org/10.1002/ajh.25822.

19. Wang $\mathrm{L}, \mathrm{He} \mathrm{W}, \mathrm{Yu} \mathrm{X}, \mathrm{Hu} \mathrm{D}, \mathrm{Bao} \mathrm{M}$, Liu H, et al. Coronavirus disease 2019 in elderly patients: characteristics and prognostic factors based on 4-week follow-up. J Inf Secur. 2020. https://doi.org/ 10.1016/j.jinf.2020.03.019.

20. Cascella M, Rajnik M, Cuomo A, Dulebohn SC, Di Napoli R. Features, evaluation and treatment coronavirus (COVID-19). Source: StatPearls [Internet]. Treasure Island: StatPearls Publishing; 2020.

Publisher's Note Springer Nature remains neutral with regard to jurisdictional claims in published maps and institutional affiliations. 